\title{
Tongue diagnosis and treatment in traditional Chinese medicine for severe COVID-19: a case report
}

\author{
Keji Liang $^{1 \#}$, Xiaodan Huang ${ }^{2,3 \#}$, Hua Chen ${ }^{4 \#}$, Liling Qiu ${ }^{5}$, Yanshan Zhuang ${ }^{6}$, Chuan Zou ${ }^{2,3}$, Yunpeng Bai ${ }^{7}$, \\ Yisheng Huang ${ }^{4}$ \\ ${ }^{1}$ Department of Chinese Medicine, Maoming City people's Hospital, Maoming, China; ${ }^{2}$ Major in Nephrology, Second Clinical Medical College, \\ Guangzhou University of Chinese Medicine, Guangzhou, China; ${ }^{3}$ Department of Nephrology, Guangdong Provincial Hospital of Chinese Medicine, \\ Guangzhou, China; ${ }^{4}$ Department of Oncology, Maoming City People's Hospital, Maoming, China; ${ }^{5}$ Department of Endocrine, Zhongshan City \\ People's Hospital (Zhongshan Hospital Affiliated to Sun Yat-sen University), Zhongshan, China; ${ }^{6}$ Teaching and Research Office of Traditional \\ Chinese Medicine, Maoming Academy of Health, Maoming, China; ${ }^{7}$ Center of Scientific Research, Maoming People's Hospital, Maoming, China \\ " The authors contributed equally to this work. \\ Correspondence to: Yisheng Huang, PhD. Department of Oncology, Maoming City people's Hospital, 101 Weimin Road, Maoming 525000, China; \\ Email: hysd999@hotmail.com.
}

\begin{abstract}
As one of the most urgent public health events, coronavirus disease 2019 (COVID-19) has attracted worldwide attention. This case highlighted the importance of close coordination between Chinese medicine and western medicine in the diagnosis and treatment, as well as the need for rapid dissemination of clinical information related to patient care with this emerging infection. We reported a COVID-19 case confirmed in China and described the identification, diagnosis, clinical course, and management of the case. The patient had initial mild symptoms at presentation; it progressed to severe pneumonia on the $10^{\text {th }}$ day of onset. This cured case supplied a time series analysis of tongue characteristics found in severe COVID-19. Chinese medicine formulae were tweaked by tongue characteristics, which include tongue color, fur thickness, and fur color. Tongue images were obtained every two days, and the changes were firmly related to the progression of COVID-19. These tongue characteristics could be used as effective, non-intrusive indices for the distinct stages of COVID-19 stages. Our study was the first time tongue diagnosis was applied in time series analysis of the progression of COVID-19 disease. We found that tongue color, fur thickness, and fur color were closely related to the progression of COVID-19 by analyzing various tongue images obtained regularly. Based on this success, we will further apply tongue diagnosis to tongue characteristics of COVID-19 patients to help limit the risk of COVID-19.
\end{abstract}

Keywords: Coronavirus disease 2019 (COVID-19); severe pneumonia; tongue diagnosis; traditional Chinese medicine (TCM); case report

Submitted May 29, 2020. Accepted for publication Jul 14, 2020.

doi: 10.21037/apm-20-1330

View this article at: http://dx.doi.org/10.21037/apm-20-1330

\section{Introduction}

The coronavirus disease 2019 (COVID-19) is not only fast but also lethal, and has become a global pandemic, killing hundreds of thousands of people. The main clinical symptoms were fever, dry cough and fatigue, and severe cases were complicated with acute respiratory distress syndrome. Generally, COVID-19 is an acutely resolving but potentially fatal disease with a case fatality rate of $3.4 \%$. Due to extensive alveolar damage and progressive respiratory failure, severe onset can lead to death $(1,2)$. As of February 29, 2020, there were 79,394 confirmed cases, and more than 2,838 deaths reported. The clinical courses and clinical outcomes were assessed in 52 of 710 laboratory-confirmed COVID-19 cases with a critical illness. The median age of the patients was 47 years; $41.9 \%$ of the patients were 
female. Only $1.9 \%$ of the patients had a history of direct contact with wildlife. Among nonresidents of Wuhan, $72.3 \%$ had contact with residents of Wuhan, including $31.3 \%$ who had visited the city. The median incubation period was 4 days (interquartile range, 2 to 7 days). On admission, ground-glass opacity was the most common radiologic finding on chest computed tomography (CT) (56.4\%). Thirty-five (67\%) patients had acute respiratory distress syndrome (ARDS), and 37 (71\%) patients required mechanical ventilation. A total of $32(61.5 \%)$ patients died at 28 days, and the median time from the intensive care unit (ICU) admission to death was 7 days for non-survivors (3). As of February 18, 2020, there were 1,331 confirmed cases in the Guangdong province. Among these 1,331 cases, 1,245 cases were treated by traditional Chinese medicine (TCM), with a participation rate of $93.54 \%$. Among these 1,245 cases, 913 cases were treated by TCM decoction alone, 177 cases were treated by TCM alone, and 155 cases were treated by TCM decoction in combination with TCM. Among the confirmed cases, 448 cases were cured, and 660 cases improved. (http://gdio.southcn.com/g/202002/19/content_190375424.htm) COVID-19 belongs to the category of "plague" in traditional Chinese medicine, which can be named "lung plague". Traditional Chinese medicine treatment has the advantages of emergency, practicality and effectiveness. For different stages and types of disease, it can improve symptoms and imaging in a short time, shorten the course of treatment and reduce the occurrence of severe diseases. However, no case treated by Chinese medicine has been reported so far due to barely accessible information acquired from a skilled practitioner. In this study, we investigated the characteristics of tongue diagnosis in a patient who survived a severe ARDS with COVID-19 by treatment with Chinese medicine in combination with western medicine. The patient belongs to the type of phlegm-heat obstructing the lung and the combination of toxin and blood stasis. The results of this study will contribute to the understanding of COVID-19 and clinical prevention and treatment strategies. Because COVID-19 is a little-known disease, we want to know it in different ways. Tongue diagnosis is a traditional diagnostic method of traditional Chinese medicine, which is simple, practical, non-invasive and rapid. We hope that through such a typical case, it can be used as a reference for the diagnosis and treatment of this kind of disease.

TCM based on holistic and systematic ideas is different from western medicine based on reductionism (4). TCM is a complete medical system and plays an indispensable role in medical care in China. Many patients and scientists believe that TCM is effective. Sometimes the palliative effect can be achieved by TCM when western medicine fails or are not available, especially for common diseases such as flu and allergies, or chronic diseases and functional disorders such as migraine and osteoarthritis that western medicine cannot relieve pain in patients.

Instead of "micro" level laboratory tests, the basis of TCM diagnostics is the overall observation of human symptoms. There are four diagnosis methods in TCM: looking, listening, asking, and feeling the pulse. In the clinical diagnosis of TCM, tongue diagnosis is an important part, which is the necessary process of non-invasive assessment of patients' viscera. It is a way to understand the physiological function and pathological changes of the human body by observing the changes in tongue color, and then conduct disease diagnosis. The traditional tongue diagnosis was evaluated by visual inspection. In this study, by observing tongue characteristics (namely color, fur thickness, and fur color) of tongue images obtained every two days, the results showed that changes in tongue characteristics were closely associated with disease progression. These characteristics could be used as effective, non-invasive indicators of distinct stages of COVID-19 progression. A yellow tongue coating is one of the classic clinical signs used to evaluate the disease in TCM.

We present the following case in accordance with the CARE reporting checklist (available at http://dx.doi. org/10.21037/apm-20-1330).

\section{Case presentation}

A 41-year-old man was admitted to a fever clinic on January 27,2020 , with symptoms of fever, chills, cough, fatigue, and shortness of breath. On January 22, 2020, he traveled from Henan to Guangzhou via Wuhan by a high-speed train and then to Xinyi by a bus. January 23, 2020, was the first day of his onset, with initial symptoms of mild chills and dry cough, but he did not visit a doctor but stayed at home until January 27, 2020 (Figure 1). The CT image of this man four times is shown in Figure 2. He presented to an urgent care clinic in Xinyi County, Maoming, with a 5-day history of cough and subjective fever. Chest computer tomography (CT) showed multiple patchy shadows in both lungs, consistent with COVID-19 changes in the right upper and lower lungs and the left lower lung (Figure $2 A$ ), and a throat swab sample was obtained. On January 29, 2020 (day 7 of illness), the Maoming Centers for Disease Control (CDC) 


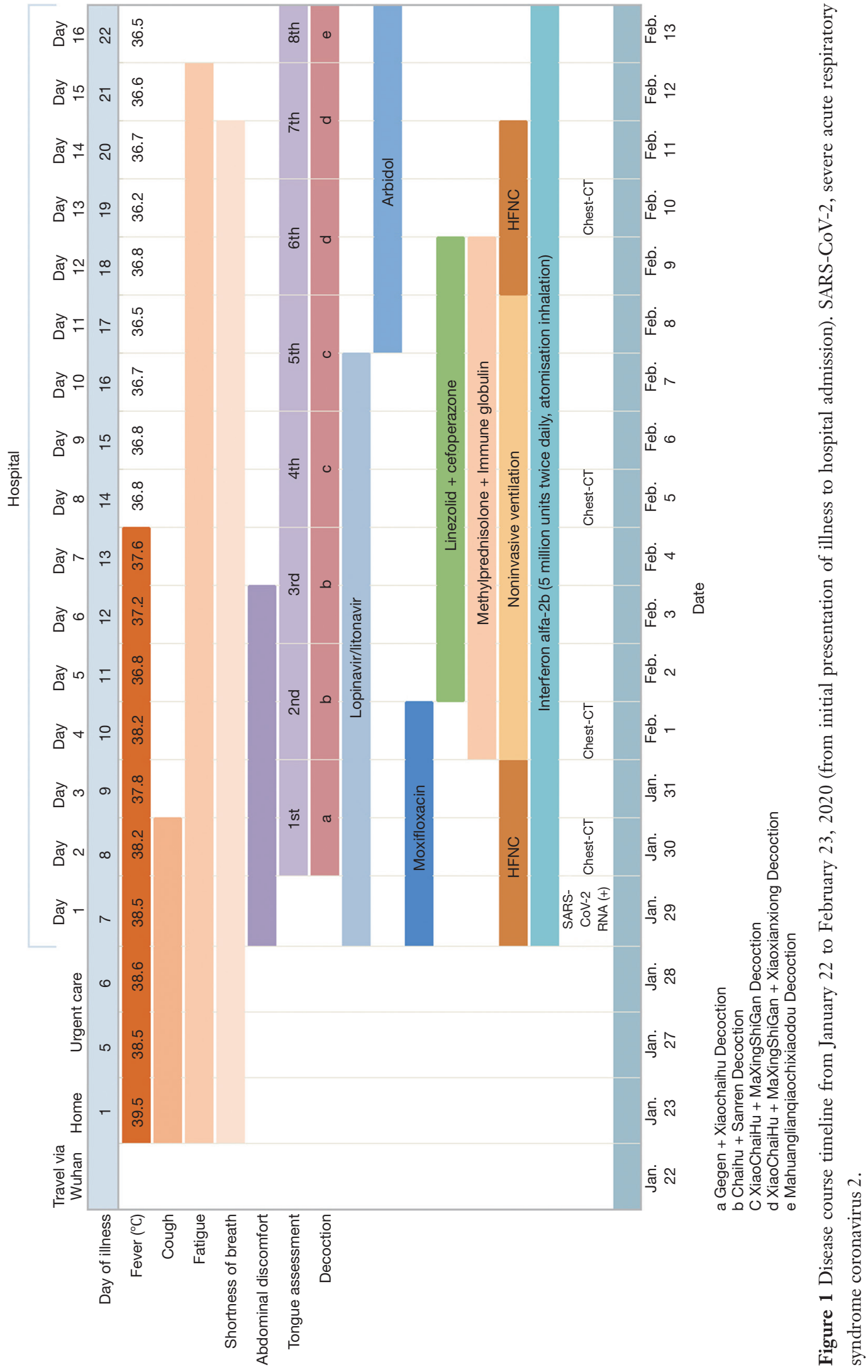



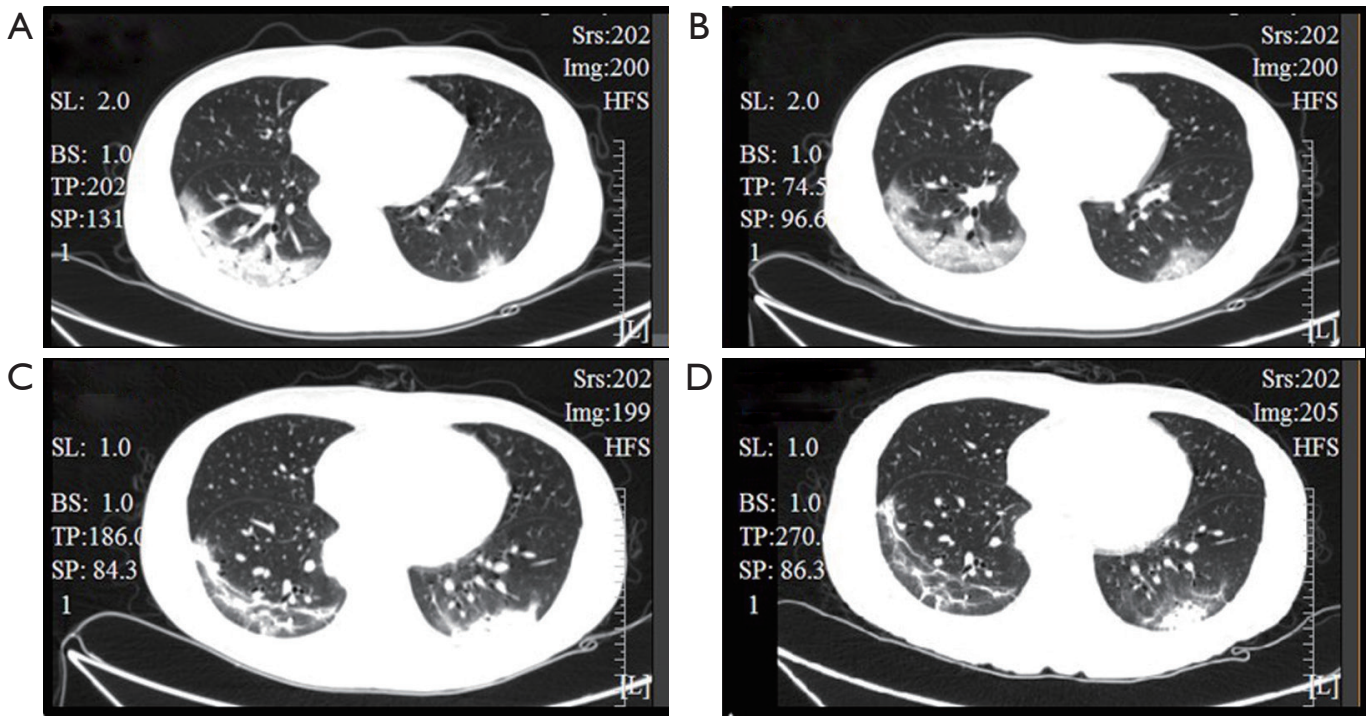

Figure 2 Chest-CT manifestations of disease timeline. (A) Multiple patchy shadows in both lungs; (B) progressive infiltration and diffusion gridding shadows in both lungs; (C) the left lower lung lesions were increased, enlarged, and combined, and the right upper and lower lung lesions were slightly absorbed; (D) absorption of bilateral lung lesions.

confirmed that the patient had COVID-19 by reverse realtime PCR assay. Written informed consent was obtained from the patient for publication of this Case report and any accompanying images. All procedures performed in studies involving human participants were in accordance with the ethical standards of the institutional and/or national research committee(s) and with the Helsinki Declaration (as revised in 2013).

Immediate admission to the isolation ward and supplemental oxygen via a mask [high-flow cannula oxygen therapy (HFNC) $(50 \%$ concentration, $40 \mathrm{~L} / \mathrm{min})]$ were conducted. Aerosol inhalation of interferon $\alpha-2 b$ (5 million units twice a day), lopinavir plus ritonavir $(500 \mathrm{mg}$ twice a day), and oseltamivir (75 mg twice a day orally) was given for antiviral therapy; moxifloxacin $(0.4 \mathrm{~g}$ once a day, intravenous injection) for secondary infection prevention. On the second day after admission, the patient was attended by a skilled TCM practitioner for the first time, and he assessed the tongue diagnosis (Figure 3, Table 1). $\mathrm{He}$ received TCM decoction (Gegeng with Xiaocaihu, daily). On February 01, 2020 (day 10 of illness), the disease had progressed. The patient developed severe dyspnea and was transferred to the ICU. Methylprednisolone (40 $\mathrm{mg}$ twice a day, intravenous injection) was administered to attenuate lung inflammation considering the severe shortness of breath and hypoxemia. Laboratory test results are listed in Figure 4. After receiving medication, his body temperature reduced from 39.0 to $36.4^{\circ} \mathrm{C}$. However, his cough, dyspnea, and fatigue did not improve. A chest CT scan showed progressive infiltrate and diffuse gridding shadows in both lungs (Figure $2 B$ ). He had received non-invasive ventilator support in the ICU; therefore, he received HFNC $(60 \%$ concentration, $50 \mathrm{~L} / \mathrm{min}$ ). At that time, the patient was attended by a skilled TCM practitioner for the second time (Figure 3, Table 1), and he received another decoction (Chaihu + Sanren, daily). On the $12^{\text {th }}$ day of onset, the patient showed symptomatic improvement with oxygen saturation above $95 \%$. The patient was attended by a skilled TCM practitioner for the third time (Figure 3, Table 1), and he received the third decoction (Chaihu + Sanren, daily). On the $14^{\text {th }}$ day of onset, his hypoxemia and shortness of breath improved. The patient was attended by a skilled TCM practitioner for the fourth time (Figure 3, Table 1), and he received another decoction (Xiaochaihu + Maxingshigan). A chest CT scan showed that the left lower lung lesions had increased, enlarged, and combined, and the right upper and lower lung lesions were slightly absorbed (Figure 2C). The oxygenation index rose from 195 to 325 on February 1, 2020 (Figure 5). On day 16 of illness, the symptom of dyspnea continued to improve (Figure 5). The patient was transferred out of the ICU. The patient stopped using lopinavir and ritonavir, and he received antiviral therapy with abidol and strengthened albumin infusion to correct the low protein level in blood. The patient was attended 


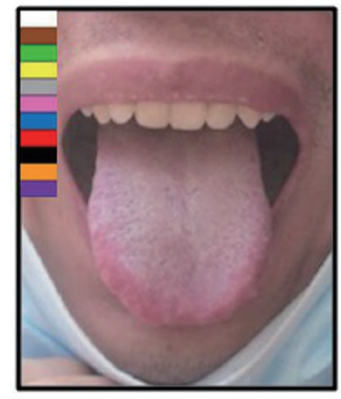

Dec. 30/ Ist

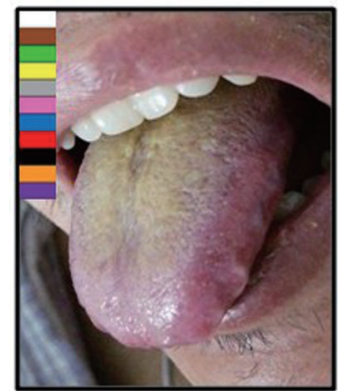

Feb. 7/5th

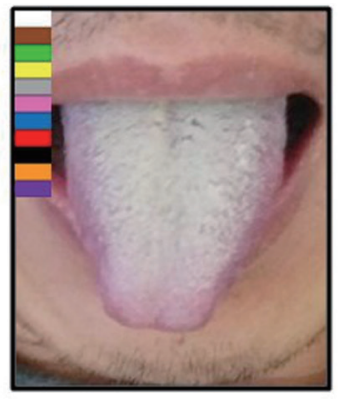

Feb. 1/2nd

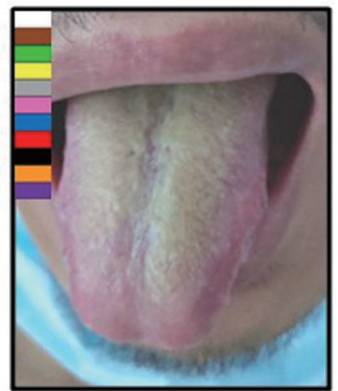

Feb. 9/6th

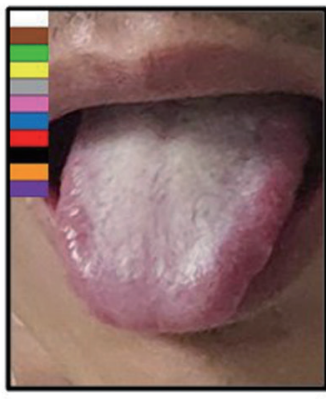

Feb. 3/3rd

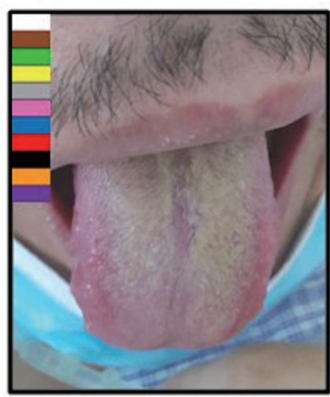

Feb. 11/7th

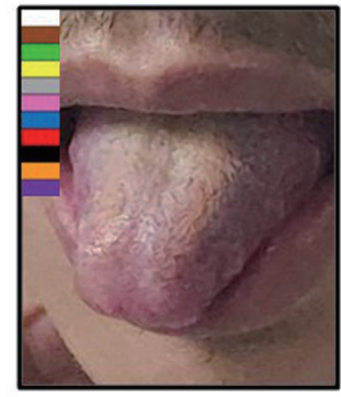

Feb. $5 / 4$ th

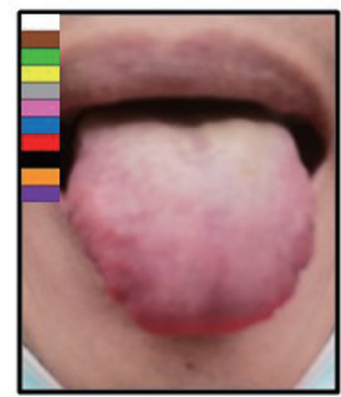

Feb. 13/8th

Figure 3 Tongue diagnosis at distinct stages of disease (1st-8th).

Table 1 Tongue characteristics

\begin{tabular}{|c|c|c|c|c|c|c|}
\hline Date & \multicolumn{2}{|c|}{ Tongue characteristics } & \multicolumn{4}{|c|}{ Fur characteristics } \\
\hline $2020-1-30$ & Moderate & Pink-red & White & Thin & Uniform & Thin \\
\hline $2020-2-1$ & Moderate & Pink-red & White & Thick & Uniform & Thick \\
\hline $2020-2-3$ & Moderate & Pink-red & White & Thick area reduced & Uniform & Thick area reduced \\
\hline $2020-2-7$ & Moderate & Pink-red & Yellow & Thick area reduced & Uneven & Thick area reduced \\
\hline $2020-2-9$ & Moderate & Pink-red & White-yellow & Less-thick & Uneven & Less-thick \\
\hline $2020-2-11$ & Moderate & Pink-red & White-yellow & Less-thick & Uneven & Less-thick \\
\hline $2020-2-13$ & Moderate & Red & White-yellow & Thin & Uniform & Thin \\
\hline
\end{tabular}

by a skilled TCM practitioner for the fifth time (Figure 3, Table 1), and he received another decoction (XiaoChaiHu + Maxingshigan + Xiaoxianxiong). On days 18, 20, and 22 of illness, the patient was attended by a skilled TCM practitioner three times (Figure 3, Table 1), and he received decoction. His symptom of dyspnea continued to improve. A chest $C T$ scan showed that the bilateral lung lesions were absorbed more than before (Figure 2D) on February 10, 2020. Finally, the patient was cured and discharged from the hospital on February 13, 2020.

\section{Discussion and conclusions}

Conventional methods work poorly in treating COVID-19, and as few virus-specific antigens have been found, no effective virus vaccine has been developed to combat COVID-19. At present, anti-virus therapy, complaint management, and maintenance therapy are the main 


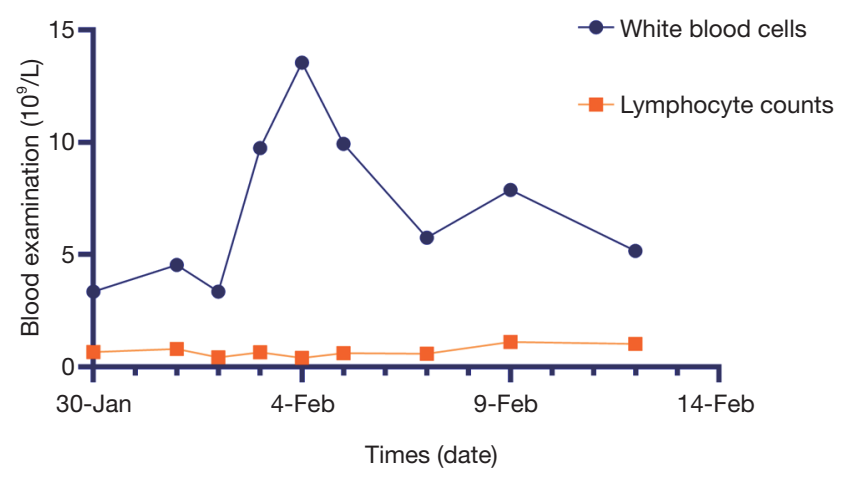

Figure 4 Lymphopenia is a common feature in patients with COVID-19 on laboratory tests.

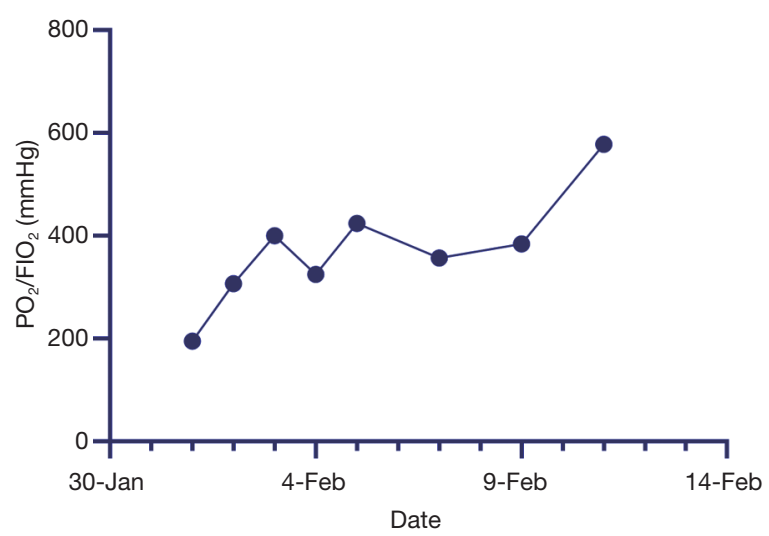

Figure 5 Change of oxygenation index: the oxygenation index rose to 325 from 195 on February 1. On day 16 of illness, the symptom of dyspnea continues to improve.

methods in modern medicine. Unfortunately, conventional anti-virus drugs, such as lopinavir plus ritonavir and abidol, do not have any effect on COVID-19. On the contrary, current evidence showed that TCM plays a vital role in controlling COVID-19, as it shortens the length of the disease and its severity; namely, it reduces the incidence of severe pneumonia and the mortality in China. Recently, TCM formulae for the treatment of COVID-19 have been approved by the National Health Commission. TCM can increase the function of the immune system and strengthen the anti-disease ability of the human body. Additionally, some decoctions, such as Xiaochaihu and Maxingshigan, have shown antiviral and antibacterial effects in the field of pharmacology of TCM. Therefore, TCM not only plays a vital role in predicting the development of the disease but also has a unique curative effect in treating COVID-19.
In long-term clinical practice, a set of unique diagnosis and treatment system has been formed according to TCM theory. The core of TCM diagnosis is "syndrome differentiation and treatment based on looking, listening, asking, and feeling the pulse." Looking ranks first among the four diagnosis methods, and tongue diagnosis is an important procedure of it. Tongue diagnosis is one of the special diagnostic methods used in Chinese medicine. The appearance of the tongue is an important index to evaluate the physiological and pathological changes of viscera (5), and all the information of the tongue is obtained by doctors' naked eyes. The diagnosis depends heavily on personal clinical experience, and different TCM experts will infer different conclusions. Some studies have shown that tongue diagnosis is crucial to clinical prognosis and treatment (6-8). The tongue is connected to the viscera through meridians. Therefore, the viscera, qi, blood, and fluid conditions, as well as the disease degree and disease progression, can be seen from the tongue. Tongue diagnosis, including shape, color, fur color, fur thickness, and other characteristics (9), reveals the conditions of organs, nature of diseases, and changes in pathogens. For example, the red tongue is closely related to the fire-heat and the yin deficiency, while the pale white tongue is related to the phlegm-dampness. According to the TCM theory, the red tongue is intricately linked to the heat pattern, with corresponding spiking fever. Thin fur is associated with the Wind and fire-heat pattern, while thick fur is usually linked to the phlegm-dampness and blood stasis patterns (5). COVID-19 belongs to the "plague" in the field of TCM. Plague attaches immense importance to tongue inspection and pulse detection. Changes in the tongue image can guide the progress of the disease. After admission, the tongue became dark red and dry, and the tongue coating turned yellow and thick. At the same time, the tongue picture changed, the condition became worse, and the patient needed to be transferred to ICU monitoring and treatment. The patient's tongue turned dark red, which indicated that the circulation of the tip of the tongue and the peripheral circulation became reduced, the blood flow rate slowed down, and the metabolism slowed down. Therefore, the ability of hemoglobin to carry oxygen decreased, and the elimination of toxins slowed down, so the patient had difficulty breathing.

Traditional tongue diagnosis inevitably has limitations, which is due to the practitioners' skills and environmental factors. This method is mainly conducted by doctors with visual observation, description language, and empirical discrimination. Therefore, personal experience and 
knowledge play an essential role in clinical skills involves tongue diagnosis. Besides, environmental factors also significantly affect the results of tongue diagnosis. The results are less reproducible for TCM because of the lack of standard evaluation criteria. According to a study on tongue diagnosis, an agreement among doctors (even the well-trained TCM doctors) is still shown to be reduced. So, objective tongue diagnosis criteria are necessary to set up. Standard operating processes and detailed guidelines for tongue diagnosis are proposed to improve the diagnosis consistency among TCM clinicians (10). Thus, this study shows the potential usefulness of tongue diagnosis for treatment progress in patients with COVID-19, whether it is for TCM intervention or conventional medical intervention. One of the strengths of TCM is that it is efficient; hence, it can be used in the entire process of disease. In some challenging cases, diagnosis becomes extremely difficult, and nucleic acid detection of COVID-19 needs to be conducted many times to obtain a precise diagnosis. Currently, how to start treatment early becomes particularly important, rather than waiting for the development of the disease and missing the opportunity for treatment.

In conclusion, this was the first application of tongue diagnosis in time series analysis of the progression of COVID-19 disease. We found that tongue color, fur thickness, and fur color were closely related to the progression of COVID-19 by analyzing various tongue images obtained regularly. Based on this success, we will further apply tongue diagnosis to tongue characteristics of COVID-19 patients to help limit the risk of COVID-19.

\section{Acknowledgments}

We are incredibly grateful for the technical support of Guangzhou Yujia Biotechnology Co., Ltd.

Funding: The study was supported by the High-level Hospital Construction Research Project of Maoming People's Hospital, the Major project of Zhongshan social public welfare science and technology research project (medical and health, 2016B1012), China Postdoctoral Foundation (Project No.: 2018m633286), and Traditional Chinese Medicine Bureau Of Guangdong Province (Project No.: 2020ZYYJ20).

\section{Footnote}

Reporting Checklist: The authors have completed the CARE reporting checklist. Available at http://dx.doi.org/10.21037/ apm-20-1330

Conflicts of Interest: All authors have completed the ICMJE uniform disclosure form (available at http://dx.doi. org/10.21037/apm-20-1330). The authors have no conflicts of interest to declare.

Etbical Statement: The authors are accountable for all aspects of the work in ensuring that questions related to the accuracy or integrity of any part of the work are appropriately investigated and resolved. All procedures performed in studies involving human participants were in accordance with the ethical standards of the institutional and/or national research committee(s) and with the Helsinki Declaration (as revised in 2013). Written informed consent was obtained from the patient for publication of this Case report and any accompanying images.

Open Access Statement: This is an Open Access article distributed in accordance with the Creative Commons Attribution-NonCommercial-NoDerivs 4.0 International License (CC BY-NC-ND 4.0), which permits the noncommercial replication and distribution of the article with the strict proviso that no changes or edits are made and the original work is properly cited (including links to both the formal publication through the relevant DOI and the license). See: https://creativecommons.org/licenses/by-nc-nd/4.0/.

\section{References}

1. Wu F, Zhao S, Yu B, et al. A new coronavirus associated with human respiratory disease in China. Nature 2020;579:265-9.

2. Huang C, Wang Y, Li X, et al. Clinical features of patients infected with 2019 novel coronavirus in Wuhan, China. Lancet 2020;395:497-506.

3. Yang $\mathrm{X}, \mathrm{Yu} \mathrm{Y}, \mathrm{Xu} \mathrm{J}$, et al. Clinical course and outcomes of critically ill patients with SARS-CoV-2 pneumonia in Wuhan, China: a single-centered, retrospective, observational study. Lancet Respir Med 2020;8:475-81.

4. You M, Ge L, Li GZ, et al. A TCM Platform for Maters' Experience Sharing, International Joint Conference on Bioinformatics, Systems Biology and Intelligent Computing (IJCBS09) Washington, DC, USA: IEEE Computer Society; 2009:388-91.

5. Anastasi JK, Currie LM, Kim GH. Understanding diagnostic reasoning in TCM practice: tongue diagnosis. 
Altern Ther Health Med 2009;15:18-28.

6. Jiang $\mathrm{M}, \mathrm{Zha} \mathrm{Q}, \mathrm{Lu} \mathrm{C}$, et al. Association between tongue appearance in Traditional Chinese Medicine and effective response in treatment of rheumatoid arthritis. Complement Ther Med 2011;19:115-21.

7. He Y, Lu A, Zha Y, et al. Differential effect on symptoms treated with traditional Chinese medicine and western combination therapy in RA patients. Complement Ther Med 2008;16:206-11.

8. Jiang WY. Therapeutic wisdom in traditional Chinese medicine: a perspective from modern science. Trends

Cite this article as: Liang $\mathrm{K}$, Huang $\mathrm{X}$, Chen $\mathrm{H}$, Qiu L, Zhuang Y, Zou C, Bai Y, Huang Y. Tongue diagnosis and treatment in traditional Chinese medicine for severe COVID-19: a case report. Ann Palliat Med 2020;9(4):2400-2407. doi: 10.21037/apm-20-1330
Pharmacol Sci 2005;26:558-63.

9. Lo LC, Chiang JY, Cheng TL, et al. Visual agreement analyses of traditional chinese medicine: a multipledimensional scaling approach. Evid Based Complement Alternat Med 2012;2012:516473.

10. Liao PY, Hsu PC, Chen JM, et al. Diabetes with pyogenic liver abscess--A perspective on tongue assessment in traditional Chinese medicine. Complement Ther Med 2014;22:341-8.

(English Language Editor: J. Chapnick) 